\title{
28 Research Square \\ Phyto Mediated Silver Nanoparticle Synthesis Coated Fabrics Towards Antibacterial Potential
}

\section{Nivedhitha Kabeerdass}

Muthayammal College of Arts and Science

\section{Karthikeyan Murugesan}

Quest International University Perak

\section{Srinivasan Ramasamy}

Tamilnadu State council for Science \& Technology, Chennai

Maghimaa Mathanmohun ( $\nabla$ mmaghimaa@gmail.com )

Muthayammal College of Arts \& Science, Rasipuram, Namakkal DT. Tamilnadu. India

https://orcid.org/0000-0002-9043-435X

\section{Research Article}

Keywords: Sore, plant extract, Antibacterial, SEM with EDX, HR-TEM, Fabrics.

Posted Date: August 20th, 2021

DOl: https://doi.org/10.21203/rs.3.rs-703040/v1

License: (c) (i) This work is licensed under a Creative Commons Attribution 4.0 International License. Read Full License 


\section{Abstract}

Sore repairing since occurrence up to dry skin formation (scar) is a precious process gifted by nature. Immunology takes a predominant part in healing. A sore which is sustained just now, within minutes/hours is usually ignored till its uninterrupted scar formation. When is delayed that persists for many days, many weeks, more months, even for years is keenly noticed and is seeking secondary healing which is donated by medical men. Uninterrupted natural healing is usually processed by the immune system of the host when healing is delayed then comes interruption of the medical experts with the inclusive of medicines such as synthetic chemicals, phytotherapy, micro nano particles mingled medicines, botanical flora yield Phyto metal micro therapies which going for updated management like $3 \mathrm{D}$ bioprinting is in practice. An array of twenty exudates of nasal, pus, urine, stool, and blood are collected from the diverse age group of sex in various wards of SOTS (such as ICU, CCU, maternity, and casualty) from the Hospitals of Madurai. After isolating from other commensals its phenotypic character is studied through gram staining and biochemical tests. To get rid of their pathogenicity a cheap, costeffective, eco-friendly potential medicinal ingredients possessed Amaranthaceae family yielded Alternanthera sessilis mediated fabrics coated silver nanoparticles were synthesized. In the case of MIC of AS-AgNPs against isolates zone of inhibition of the $E$. coli is $16 \mathrm{~mm}>S$. aureus is $15 \mathrm{~mm}$ at $60 \mu \mathrm{g}$. But in the case of MIC of fabrics against isolates the $E$. coli and $K$. oxytoca show susceptibility of $19 \mathrm{~mm}$ in mixed cotton. In a confirmation study of AS-AgNPs in FTIR, a sharp band is observed between 1500 $500 \mathrm{~cm}-1$, in SEM with EDAX a graph was observed between 2.6-3.9 and black white crystals measured at $200 \mathrm{~nm}$ in HR-TEM. Nanotechnology conjoined with herbal therapeutics provides a promising solution for wound management strategies.

\section{Introduction}

Sustained injury is an incidental or accidental event. The texture of the pellicle is interrupted which is an opened one at the moment of insufficient sterilization with ignorance due to the accumulation of pathogenic microbes that acquire pathogenicity [1]. Longing for preparations with efficacy exploring synonym botanical floral metallic nano therapies. Alternanthera sessilis possessing medicinal ingredients like alkaloids, flavonoids, tannins, antioxidants, steroids which are all should be utilized like medicinal therapeutic chemicals mingled with silver nanoparticles in such a way the target filled is enriched with static and cidal expectations due to their physical, chemical, organic, inorganic and morphological structures aiding the field is pooled satisfactorily to gain fast and complete healing [2].The trade secrecy of utilization of this sap or latex or internal medical ingredients by soiling with the textile fabrics and in the post SEM, TEM treatment after getting a conclusion the sedimented nanomaterials augment with a healing character possessing valuable inclusive always ready to explore their extraordinary efficacy mingled phytotherapy to provide an unexpected destructive power towards the invisible sequelae causing antigens by getting an incredible title named sensitive structures towards MDR, XDR and PDR antigens. Not only their play involved in the pre and acting healing mechanisms but also an important and expected beneficial result which will be prolonged up to the end of the life comparatively a longer process 
than the same of the pre and acting mechanisms that is the attainment of non-keloidal smooth rejuvenation by epithelialization replacement over the acquired pucker [3]. In the repairing of the sustained sore, the post healing period is a very sensitive and important period when compared with the period of healing that is the pre scar formation period due to the vast and fast micro nanomaterials saturating the battlefield is reflected as a rejuvenating epithelization replacement by smooth healthy cells reappearance such that morphologically or appearance of even though sustaining post-lesion scar due to the micro nano particles reactionary make the obtained scar as visually a scar-free status. Why we are insisting here regarding the occurrence of rejuvenating reepithelization because all the therapeutic veins experienced towards the sequelae of wound healing are not up to our expectations (keloids). The botanical floral Phyto therapies whenever mingled with metal nanoparticles the result in the acquire of the explained sequelae (keloid) towards the recovery of the sustained puckers are minimized sometimes resulting in nil sequelae. So due to the Phyto micro nanoparticles, rich therapies gain us an incredible benefit that is even after wound repair a permanent and cosmetically the worst hazard (keloid) which is a life long inconvenience for which instrumental manipulation facing can be neglected by botanical floral Phyto micro-nano therapies like Alternanthera sessilis + silver nanoparticles mingled compound [4].

\section{Methodology}

\subsection{Collection of pathogenic strains}

Using a dry cotton swab tip the dermis of the lesions are rubbed and placed in an inverted position in a sterile tube. Sterile cylindrical capped tubes are used to collect the first voiding sputum. The midstream urine is collected in a sterile nonporous container. An unbreakable screw cap bottle integrated with a spoon is used to collect the mucous or blood coated stools. Sterile IV needles are used to drain blood samples which are then loaded in a tube covered with EDTA to avoid clotting [5]. Name, age, sex, color, and texture of the sample using waterproof pen legibly noted on the sealed sample and placed them in an icebox and shifted to the laboratory within $6 \mathrm{hrs}$. All these samples are collected from different wards of various hospitals and laboratories in Madurai. Pre-collection techniques are followed properly to isolate the suspicious pathogen and cross-contamination is prevented by wearing gloves, a mask lab coat, and a head cap [6].

\subsection{Isolation and screening}

MacConkey agar, Nutrient agar, blood agar, and mannitol salt agar, are prepared in appropriate concentration than using an incinerated loop or sterile dry cotton swab gently touch the surface of the exudates in the collection tube than by zig-zag, perpendicular, and quadrant method of streaking inoculation done in their corresponding labeled plate [7]. Texture, color, size of each colony is noted after 12-24 hrs of incubation then after incineration of the loop a colony is rubbed on the slide and showed in the flame for smear preparation. Then the smear is wetted by crystal violet, mordant, acetone, and at last safranin pro a minute and cleansed in water, air dried, and observed under the microscope to observe whether it is a gram-positive or gram-negative bacteria. IMVIC, oxidase, urease, mannitol, and citrate are 
the confirmatory biochemical test done to identify the above isolates which show a color change by adding reagents like methyl red Kovacs reagent, glucose broth, etc [8].

\subsection{Floral collections}

A cost-effective, viridescent, stalkless, perennial herb is plucked in the ponds, and canals from the nearby villages of Rasipuram [9]. Then surface sterilization is done by rinsing in water and separated the affected leaf. Then clean, unaffected leaves are shadow dried, pulverized and made into fine crystals for extraction.

\subsection{AS-AgNPs synthesis}

After finding the dry weight of the fine crystals they are treated with aqua and then stirred continuously into a concentrated solution. After adding in a small beaker they are placed in a heating mantle and parameters optimization is done [10]. Then the precursor silver nitrate at a concentration of $1 \mathrm{Mm}$ is mixed gently. In the absence of light after 72 hrs a color change from straw to chocolate brown was monitored at regular intervals this indicates the presence of silver atoms in the process and finally, the collected residues from the Whatman filter paper No1 are kept in the hot air oven at a temperature of $80^{\circ} \mathrm{C}$ to remove the water droplets completely. Later those granules are stored for further standard confirmatory analysis [11].

\subsection{Antibacterial activity}

In prepared MHA plate using a sterile dry cotton swab tip touched the surface of the isolated colony gently and streaked completely all over the plate then after incineration of the cork Borell, $6 \mathrm{~mm}$ a punch was made on the plate $[12,13]$. Then all the plugs are removed aseptically using forceps or loop. Different concentrations of AS-AgNPs crude (such as $20 \mu \mathrm{g}, 40 \mu \mathrm{g}$, and $60 \mu \mathrm{g}$ ) using micropipette loaded on each well. After inoculation, the plates are placed in the upright position in the incubator for $24-48$ hrs [14].

\subsection{Fabric sensitivity test}

In the freshly prepared muller, Hinton agar plate the pathogens are spread evenly by using a sterile dry swab, and then fabrics such as cotton and mixed cotton are made into small square pieces soiled in crude of AS-AgNps placed in the plate [15]. Then a non coated AS-AgNps fabrics act as a control.

\subsection{Confirmational study of AS-AgNPs coated on fabrics}

\section{i) UV-Visible spectroscopy}

The silver nanoparticles synthesis from the phyto extract processed in a DBPEL (double-beam PerkinElmer Lambda) UV/Vis spectrometer and all the processes take place in fused silica glass cuvette under magneto stirring. 


\section{ii) FTIR}

FTIR is used to establish the functional groups, absorption, and efficiency between different components of the synthesized product is noted [16]. After preparing the sample it is placed in the infrared beam using a sample holder then after many scans are taken and its resolution, the spectral range is measured.

\section{iii) SEM with EDAX}

It is used to generate the corresponding electron from the electron detector and also generate the surface topological image of the specimen at elevated magnification [17]. Samples are prepared by cutting into thin films using diamond abrasives and mounted if the surface is rough they are scratched out using sandpaper or pumice stone and cleansed using solvents like methanol, ethanol, and acetone, and finally, thermal etching analysis is done.

The energy distribution and photon of light focused on the surface of the specimen by Energy-dispersive $\mathrm{X}$-ray spectrometers and is used to measure the metal nanoparticles qualitatively and quantitatively in the material.

\section{iv) HR-TEM}

A crystallographic structure of the image at an atomic level is recorded at high magnification and resolution with the help of a beam of electrons [18]. The sample is made into thin sections using diamond abrasives, mounted, and finally, the image in nanometer-scale against the black and white background is observed.

\section{Results}

\subsection{Isolated strains}

Among twenty strains $5 \%$ of strains isolated from ICCU are gram-negative and gram-positive organisms, $5 \%$ of strains isolated from CCU are gram-negative organisms, $5 \%$ of strains isolated from maternity and general wards are gram-negative and other commensals are shown in Figure 1. Confirmatory biochemical tests such as IMVIC, oxidase, citrate, urease, and sugar fermentation tests show positive signs to their corresponding isolates.

\subsection{AS-AgNPs synthesis}

The crude (leaf extract) was treated with $1 \mathrm{Mm}$ of the precursor (silver nitrate) and with continuous stirring it was heated in the heating mantle for a temperature of $100^{\prime} \mathrm{C}$. Then it was cooled and then the supernatant was removed and the sediment is washed several times in Milli-Q water. The obtained residue was kept in the oven to remove the water droplets present in it.

\subsection{Antibacterial activity}


In this minimal inhibitory concentration, the gram-negative bacteria like K.pneumoniae, E.coli, K.oxytoca shows an elevated zone of inhibition of $16 \mathrm{~mm}$ at $60 \mu \mathrm{g}$ concentration which indicates it is more susceptible than gram-positive organism like S.aureus shows less inhibitory action $(15 \mathrm{~mm}$ at $60 \mu \mathrm{g}$ concentration) against the AS-AgNPs is shown in table 1 and Figure 2.

\subsection{Fabric sensitivity test}

In MHA plate mixed cotton fabric soaked AS-AgNPs show more sensitivity against E.coli and K.oxytoca is $19 \mathrm{~mm}$ but in the case of gram-positive organisms, S.aureus shows less sensitivity of $17 \mathrm{~mm}$ against the fabrics. Among these two fabrics, mixed cotton fabrics are more predominant. The values tabulated are shown in table 2 and Figure 3.

\subsection{Confirmational study of AS-AgNPs coated on fabrics}

\section{i) UV- visible Spectrum}

The uppermost UV-absorbance of the synthesized AS-AgNPs is about $330 \mathrm{~nm}$ (Figure 4).

\section{ii) FTIR}

Aliphatic, aromatic compound in the aqueous extract of $A$. sessilis and also outline the presence of the phytochemistry which is revealed by its transmission peaks at $500,1000,1500$, and $2000 \mathrm{~cm}$. A band is assigned to an aqueous molecule is in the range of $3500 \mathrm{~cm}$ it is used to identify many compounds in the extract is shown in figure 5 .

\section{iii) SEM with EDAX}

A small white cottony AS-AgNPs is embedded on the fabrics with a voltage of accelerating electrons at $30 \mathrm{KV}$ and a wide of $10.27 \mathrm{~mm}$ viewed the picture at a magnification of 578Xis shown in figure 7.

In EDX the metal atom from the nanoparticles with a peak in the range of 2.6-3.9 this indication is shown in figure 7. It also reflects the presence of other metals such as curium(CK), oxygen, potassium(O, $\mathrm{K})$, and silver iodide(Agl) with their molecular and atomic mass is shown in table 3 .

\section{iv) HR-TEM}

Aspherical black small ball-shaped Ag nanoparticles coated on mixed cotton fabrics with a particle size of $200 \mathrm{~nm}$ pictures are recorded by the LCD camera in fig 6 .

\section{Discussion}

The phytochemicals of this botanical flora Alternanthera sessilis are concentrated with pharmaco healers which regulate and replace the deleted and declined naturals of homeostasis. This insinuated action triggers the rejenuvating concretion. To attain this status the swelling subsiders, the nutrient providers, the 
pain killers an unpleasant odor refreshners, the smoothening pawers, the cytopathology recovery yielders most necessitating degredatory degenerating dwellers arresting antibody shielders suppliers originality oriental owned re-epithelialization builders by terpenoids, alkaloids, flavonoids, tannins, serotonins, bradykinins, steroids and cholins (acetyl, catacolamins). Thus anti-inflammatory, anti pyritic anti oxidantic, analgesic efforts of the floral ingredients are well explored with all these. This flora with metallic microbial Ag micro particles full fills the expectations in such a way that eradicating the diseasecausing causatives by their static and cidal personalities. Practical performed by the collections of the transudates and exudates from organisms (pathogenic) crowded flora through a way of international protocols after allowing into a various sterile procedures the obtained final readily applicable media for further manipulations evidenced by gram stains, dip diffusions, SEM, TEM, HR-TEM, and FTIR reveal the following evidence. The disease management against MDR strains is treated by Phyto metal nanoparticles which have potential and unique properties against antibacterial and anti fabric sensitivity assay. In which the gram-negative organisms E.coli and K.oxytoca show a susceptibility of $16 \mathrm{~mm}$ at 60 $\mu \mathrm{g}$ concentration. In mixed cotton fabrics, gram-negative organisms like $E$. coli and K.oxytoca show a good zone of inhibition of $19 \mathrm{~mm}$ [19]. Then fabrics coated by AS-AgNPs are finally confirmed by a bandwidth of $3500 \mathrm{~cm}$ which is used to identify the other relevant aliphatic compounds coated on the fabrics. At a magnification of $578 x$ AS-AgNPs embedded on the fabrics with a voltage of $30 \mathrm{kV}$ is shown in the black and white by the LCD camera integrated into SEM. A peak elevated in the range of 2.6-3.9 along with other metals with their physical and chemical properties is elucidated in EDX. The camera inHR-TEM reflects the size of the AS-AgNPs is $200 \mathrm{~nm}$ which is coated on the fabrics like small, round black balls. This proves gram-negative is more predominant and potent than gram-positive against Phyto mediated nanoparticles agents $[20,21]$.

\section{Conclusion}

When economically important medicinal micro phytochemicals mingled with valuable silver microparticles gain the most beneficial and efficient expectational characters like target fulfilling personalities like accessing the battlefield abundant and also attain saturated soilings with most morphologically ultra micro volumed efficient ingredients (silver nanoparticles) which yield the appreciated and expected healing antibodies stature thus enhance the healing performance. These botanical floral silver nanotherapies stable their importance in the wound repairing process. This work acknowledges that the metal microparticles along with Alternanthera sessilis enhance and pave the way for scavenging free radicals because it is toxic-free, eco-friendly acts as a vehicle $[22,23]$ to treat the biofilm on the ulcer.

\section{Declarations}

\section{Acknowledgment}

The authors are thankful for the financial support from Tamilnadu State Council for Science \& Technology (TNSCST), DOTE Campus, Chennai (Science \& Technology Project: TNSCST/STP- 
PRG/AR/2018-2019), and DST-FIST Centralized laboratory, Muthayammal College of Arts \& Science, Rasipuram, Namakkal DT. Tamilnadu, India for executing this work.

\section{Funding}

Tamilnadu State Council for Science \& Technology (TNSCST), DOTE Campus, Chennai (Science \& Technology Project: TNSCST/STP-PRG/AR/2018-2019).

\section{Conflicts of interest/Competing interests}

There is no conflict of interest.

\section{Availability of data and material}

Not applicable.

Code availability (software application or custom code)

Not applicable.

\section{Authors' contributions}

Nivedhitha Kabeerdass ${ }^{1}$ Executed the research work.

Murugesan Karthikeyan ${ }^{2}$ and Srinivasan Ramasamy ${ }^{3}$, performed the instrumentation study

*Maghimaa Mathanmohun ${ }^{1}$ - Designed and Supervised the work

\section{Ethics approval}

Not applicable.

\section{Consent to participate}

Not applicable

\section{Consent for publication}

Not applicable

\section{References}

1. Yosari, S., Pontaza-Licona Ramos-Jacques, A.L., Cervantes-Chavez, J.A., Luis López-Miranda, J., Álvaro de Jesús, R.B., Maya-Cornejo, J., Rodríguez-Morales, A.L., Esparza, R., Estevez, M., Pérez, R., Hernandez-Martínez, A.R.: Alcoholic extracts from Paulownia tomentosa leaves for silver 
nanoparticles synthesis. Results Phys. 12, 1670-1679

(2019). https://doi.org/10.1016/j.rinp.2019.01.082.

2. Elumalai, D., Hemavathi, M., Deepaa, C.V., Kaleena, P.K.: Evaluation of phytosynthesised silver nanoparticles from leaf extracts of Leucas asperaand Hyptis suaveolensand their larvicidal activity against malaria, dengue and filariasis vectors. Parasite Epidemiol. Control. 2(4), 152(2017). https://doi.org/10.1016/j.parepi.2017.09.001.

3. Rocha-Rocha, O., Cortez-Valadez, M., Hernandez-Martinez, A.R., Gamez-Corrales, R., Alvarez, R.A.B., Britto-Hurtado, R., Delgado-Beleño, Y., Martinez-Nuñez, C.E., Pérez-Rodríguez, A., Arizpe-Chávez, H., Flores-Acosta, M.: Green synthesis of Ag-Cu nanoalloys using Opuntia ficus-indica. J. Electron. Mater. 46, 802 (2017). https://doi.org/10.1007/s11664-016-4942-2CAS.

4. Marulasiddeshwara, M.B., Dakshayani, S.S., Sharath Kumar, M.N., Chethana, R., Raghavendra Kumar, P., Devaraja, S.: Facile-one pot-green synthesis, antibacterial, antifungal, antioxidant and antiplatelet activities of lignin capped silver nanoparticles: a promising therapeutic agent. Mater. Sci. Eng. C Mater. Biol. Appl. 81, 182-190 (2017). https://doi.org/10.1016/j.msec.2017.07.054CAS.

5. Dakshayani, S.S., Marulasiddeshwara, M.B., Ramesh, M.N.S., Raghavendra Kumar, P., Devaraja, S.R.H.K.: Antimicrobial, anticoagulant and antiplatelet activities of green synthesized silver nanoparticles using Selaginella(Sanjeevini) plant extract. Int. J. Biol. Macromol. 131, 787-797 (2019). https://doi.org/10.1016/j.ijbiomac.2019.01.222 CAS.

6. De-Zhi, L., Hong-Da, C., Feng, B., Zhen-Xin, W.: Progress of multimodal molecular imaging technology in diagnosis of tumor. Chin. J Anal. Chem. 44, 1609-1618 (2016). https://doi.org/10.1016/S18722040(16)60966-0.

7. Nikparast, Y., Saliani, M.: synergistic effect between phyto-synthesized silver nanoparticles and ciprofloxacin antibiotic on some pathogenic bacterial strains. J. Med. Bacteriol. 7, 36-43 (2018) CAS.

8. Snega, S., Ravichandran, K., Baneto, M., Vijayakumar, S.: Simultaneous enhancement of transparent and antibacterial properties of ZnO films by suitable F doping. J. Mater. Sci. Technol. 31, 759-765 (2015). https://doi.org/10.1016/j.jmst.2015.03.001.

9. Agilan, B., Rajendra Prasad, N., Kanimozhi, G., et al. Caffeic acid inhibits chronic UVB-induced cellular proliferation through JAK-STAT3 signaling in mouse skin. Photochem Photobiol. 2016; 92:467-474.

10. Aritonang, H. F., Kamu, V. S., Ciptati, C., Onggo, D., and C. L. Radiman. “Performance of platinum nanoparticles/multiwalled carbon nanotubes/bacterial cellulose composite as anode catalyst for proton exchange membrane fuel cells," Bulletin of Chemical Reaction Engineering \& Catalysis, vol. 12, no. 2, pp. 287-292, 2017.

11. Xu X, Ho W, Zhang X, et al. Cancer nanomedicine: from targeted delivery to combination therapy. Trends Mol Med. 2015; 21:2 23-232.

12. Jeevanandam, J., Barhoum, A., Chan, YS., et al. Review on nanoparticles and nanostructured materials: history, sources, toxicity and regulations. Beilstein J Nanotechnol. 2018;9:1050-1074. 
13. Maghimaa, M., Alharbi, SA. Green synthesis of silver nanoparticles from Curcuma longa L. and coating on the cotton fabrics for antimicrobial applications and wound healing activity. Journal of Photochemistry and Photobiology B: Biology. 2020; 204:111806.

14. Balupillai A, Nagarajan RP, Ramasamy K, et al. Caffeic acid prevents UVB radiation induced photocarcinogenesis through regulation of PTEN signaling in human dermal fibroblasts and mouse skin. Toxicol Appl Pharmacol. 2018;352:87-96.

15. Vakayil R, Kabeerdass N, Srinivasan R, et al. Invitro and insilico studies on antibacterial potentials of phytochemical extracts, Materials Today: Proceedings. 2021. https://doi.org/10.1016/j.matpr.2021.05.017

16. R. Vazquez-Muñoz, B. Borrego, K. Juárez-Moreno et al., "Toxicity of silver nanoparticles in biological systems: does the complexity of biological systems matter?" Toxicology Letters, vol. 276, pp. 11-20, 2017.

17. Baharara J, Ramezani T, Divsalar A, et al. Induction of apoptosis by green synthesized gold nanoparticles through activation of caspase-3 and 9 in human cervical cancer cells. Avicenna J Med Biotechnol. 2016;8:75-83.

18. Xu X, Ho W, Zhang X, et al. Cancer nanomedicine: from targeted delivery to combination therapy. Trends Mol Med. 2015;21:223-232.

19. Rizvi SAA, Saleh AM. Applications of nanoparticle systems in drug delivery technology. Saudi Pharm J. 2018;26:64-70.

20. Kunoh T, Takeda M, Matsumoto S, et al. Green synthesis of gold nanoparticles coupled with nucleic acid oxidation. ACS Sustainable Chem Eng. 2017;6:364-373.

21. Khan I, Saeed K, Khan I. Nanoparticles: properties, applications and toxicities. Arabian J Chem. 2017.

22. Watkins $\mathrm{R}$, Wu L, Zhang $\mathrm{C}$, et al. Natural product-based nanomedicine: recent advances and issues. Int J Nanomed. 2015; 10:6055-6074.

23. Kabeerdass $\mathrm{N}$, Krishnamoorthy $\mathrm{S}$, Anbazhagan $\mathrm{M}$ et al. Screening, detection and antimicrobial susceptibility of multi-drug resistant pathogens from the clinical specimens, Materials Today: Proceedings. 2021. https://doi.org/10.1016/j.matpr.2021.05.018.

\section{Tables}

\section{Table 1-Antimicrobial sensitivity of synthesized AS-AgNPs against the isolates}




\begin{tabular}{|lllll|}
\hline S. No & Name of the organism & $\mathbf{2 0} \boldsymbol{\mu g}$ & $\mathbf{4 0} \boldsymbol{\mu g}$ & $\mathbf{6 0} \boldsymbol{\mu g}$ \\
\hline 1 & E.coli & $13 \mathrm{~mm}$ & $14 \mathrm{~mm}$ & $16 \mathrm{~mm}$ \\
\hline 2 & Staphylococcus aureus & $12 \mathrm{~mm}$ & $14 \mathrm{~mm}$ & $15 \mathrm{~mm}$ \\
\hline 3 & Pseudomonas aeruginosa & $13 \mathrm{~mm}$ & $14 \mathrm{~mm}$ & $15 \mathrm{~mm}$ \\
\hline 4 & Klebsiella pneumonia & $14 \mathrm{~mm}$ & $15 \mathrm{~mm}$ & $16 \mathrm{~mm}$ \\
\hline 5 & Klebsiella oxytoca & $13 \mathrm{~mm}$ & $15 \mathrm{~mm}$ & $16 \mathrm{~mm}$ \\
\hline 6 & Acinetobacter baumanii & $11 \mathrm{~mm}$ & $12 \mathrm{~mm}$ & $13 \mathrm{~mm}$ \\
\hline
\end{tabular}

Table -2 Antimicrobial sensitivity test of fabrics (cotton and mixed cotton) against the isolates

\begin{tabular}{|llll|}
\hline S.No. & Name of the organism & Cotton fabrics & Mixed cotton fabrics \\
\hline 1 & E.coli & 17 & 19 \\
\hline 2 & Staphylococcus aureus & 17 & 15 \\
\hline 3 & Pseudomonas aeruginosa & 17 & 16 \\
\hline 4 & Klebsiella pneumoniae & 17 & 18 \\
\hline 5 & Klebsiella oxytoca & 17 & 19 \\
\hline 6 & Acinetobacter baumanii & 11 & 13 \\
\hline
\end{tabular}

Table 3 Elements analysis of synthesized AgNPs by EDX

\begin{tabular}{|llllllllll|}
\hline Element & $\begin{array}{l}\text { Weight } \\
\%\end{array}$ & $\begin{array}{l}\text { Atomic } \\
\%\end{array}$ & $\begin{array}{l}\text { Net } \\
\text { Int. }\end{array}$ & $\begin{array}{l}\text { Error } \\
\%\end{array}$ & Kratio & Z & R & A & F \\
\hline C K & 49.22 & 57.27 & 553.40 & 6.12 & 0.3023 & 1.0280 & 0.9851 & 0.5971 & 1.0000 \\
\hline O K & 48.60 & 42.45 & 404.74 & 10.10 & 0.0926 & 0.9826 & 1.0042 & 0.1939 & 1.0000 \\
AgL & 2.18 & 0.28 & 53.01 & 5.36 & 0.0182 & 0.6677 & 1.2639 & 1.2024 & 1.0409 \\
\hline
\end{tabular}

\section{Figures}


Figure 1 ISOLATED STRAINS FROM WARDS

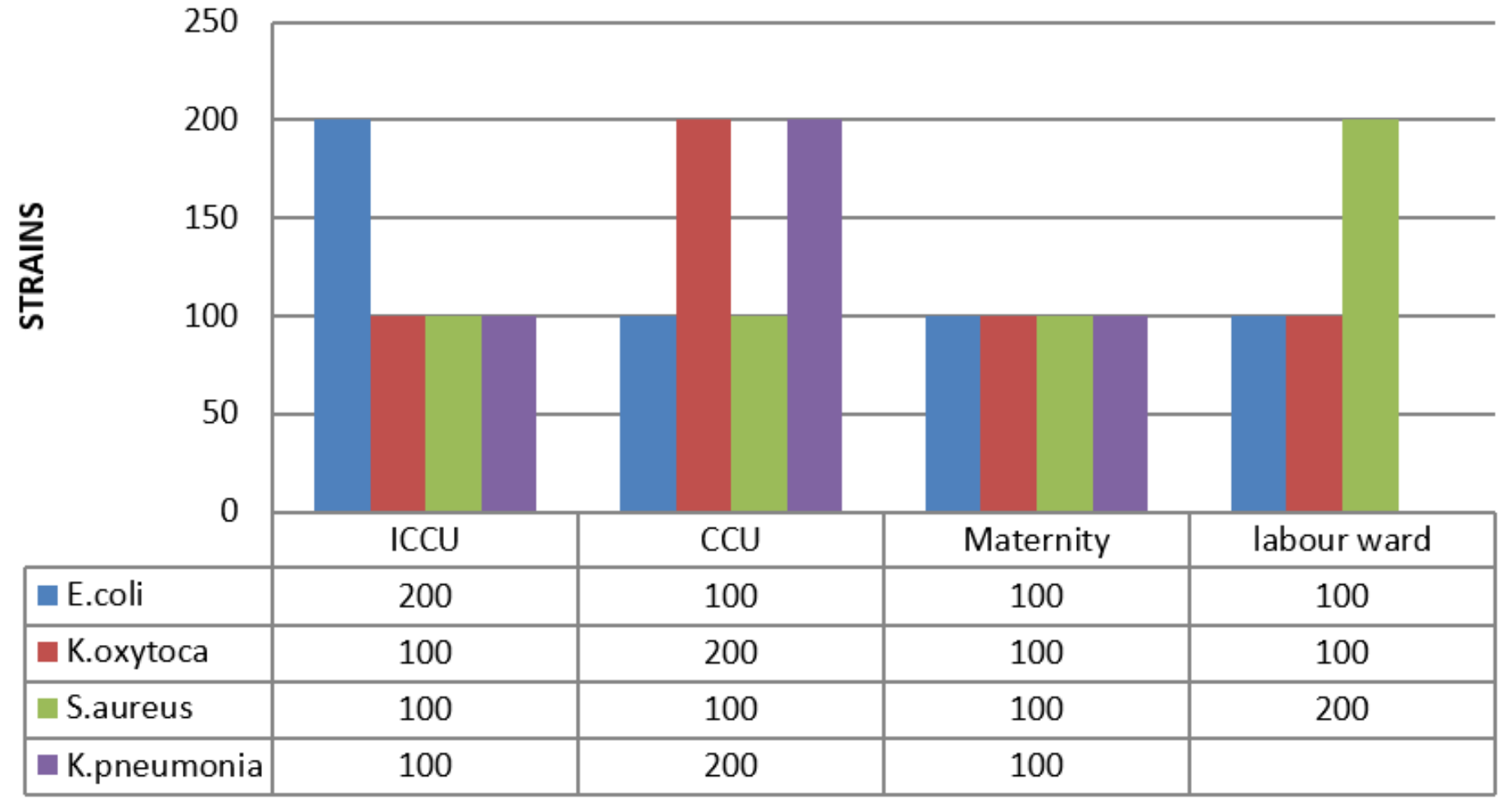

Figure 1

Isolated Strains From Wards 


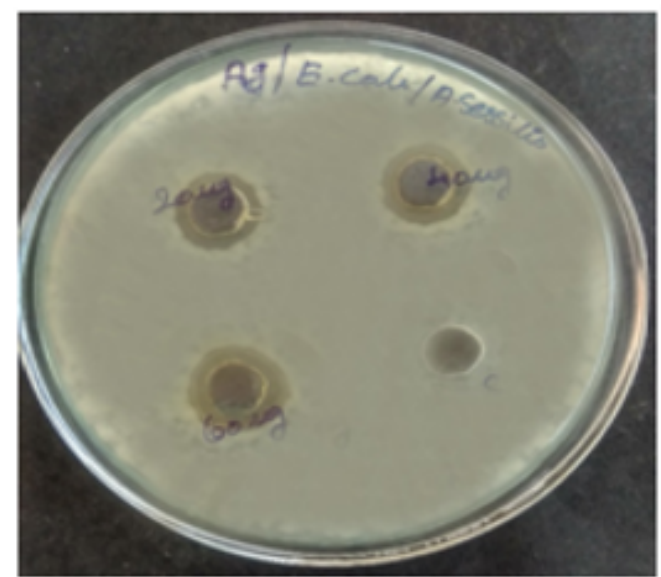

E.coli

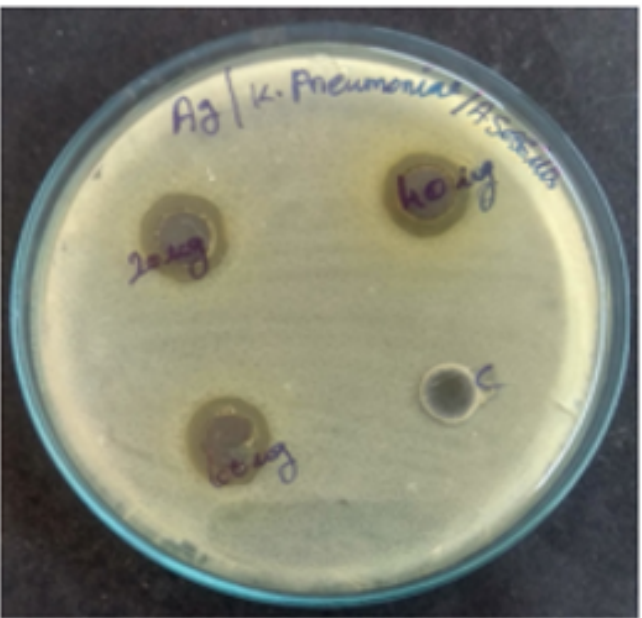

Klebsiella pneumoniae

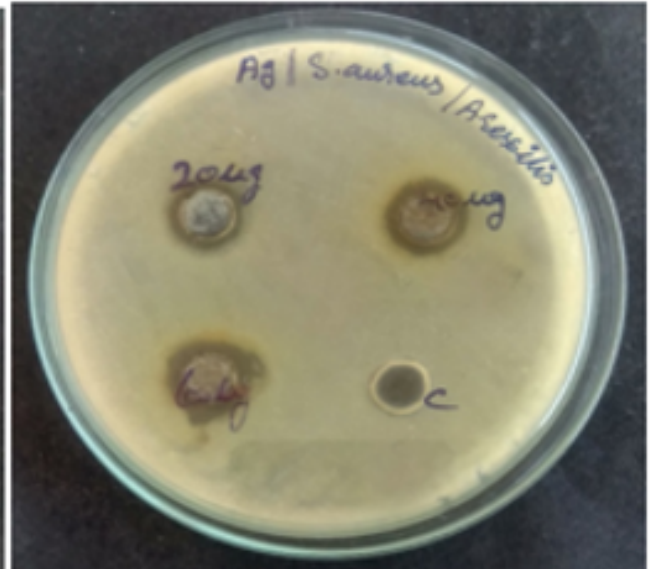

Staphylococcus aureus

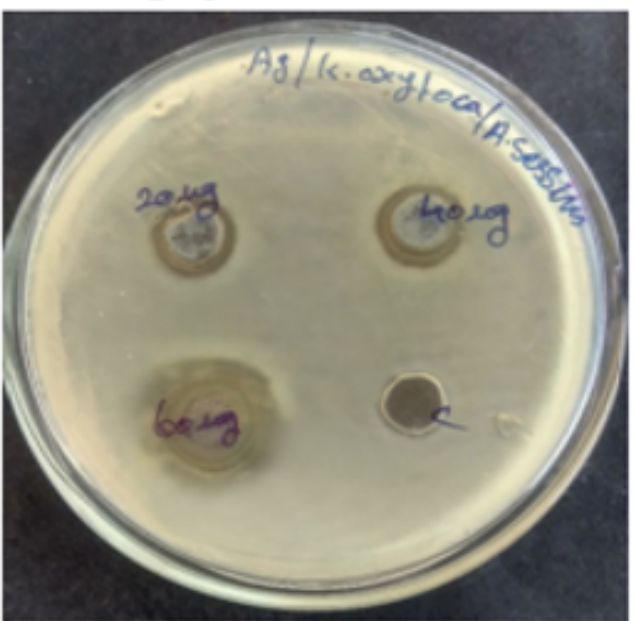

Klebsiella oxytoca

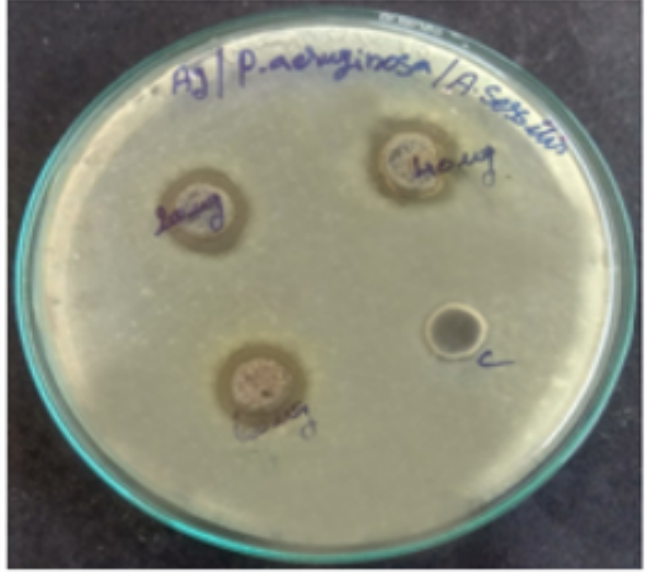

Pseudomonas aeruginosa

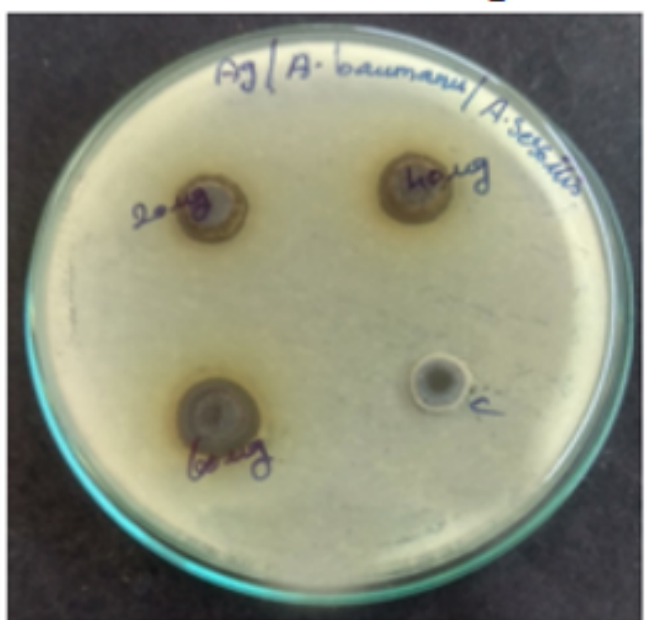

Acinetobacter baumanï

Figure 2

Antimicrobial sensitivity of synthesized AS-AgNPs against the isolates 


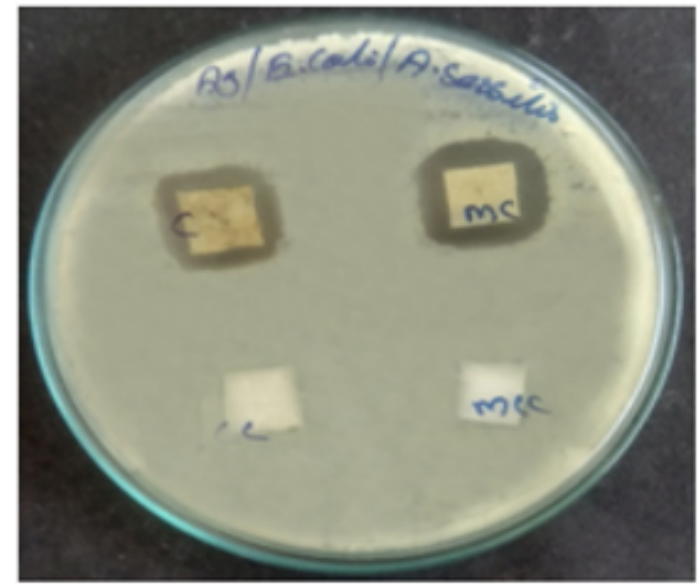

E.coli

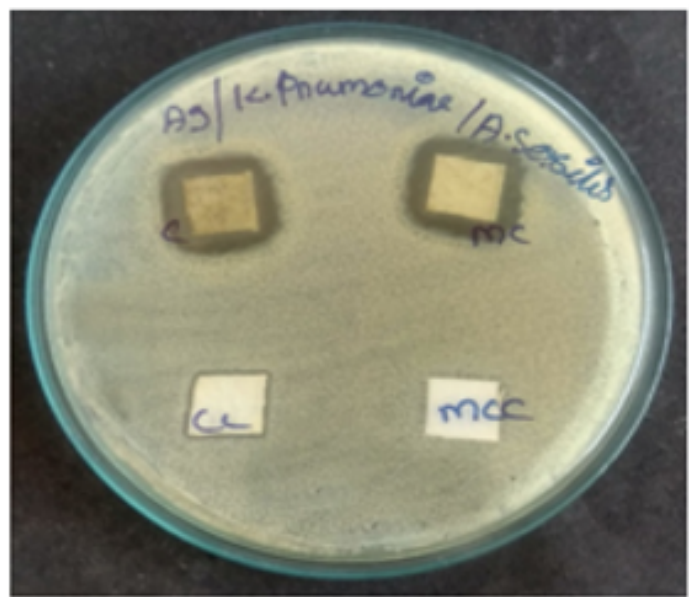

Klebsiella pneumoniae

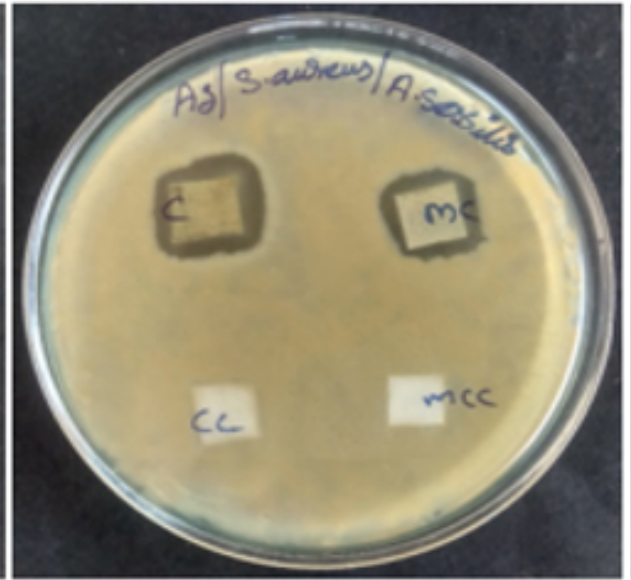

Staphylococcus aureus

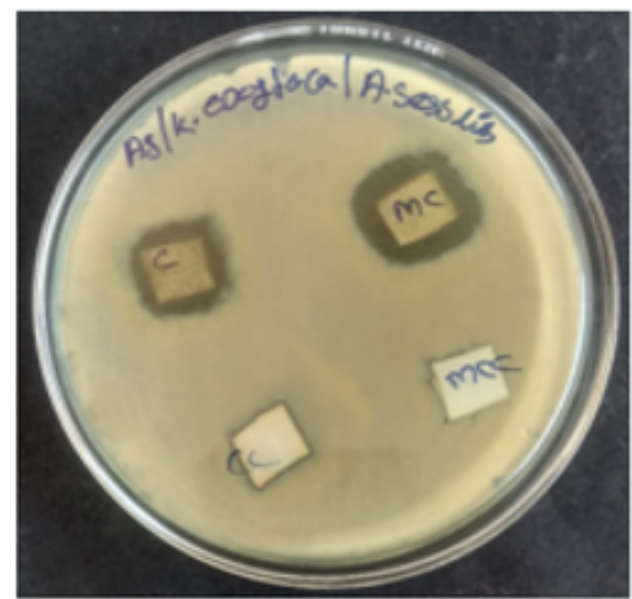

Klebsiella oxytoca

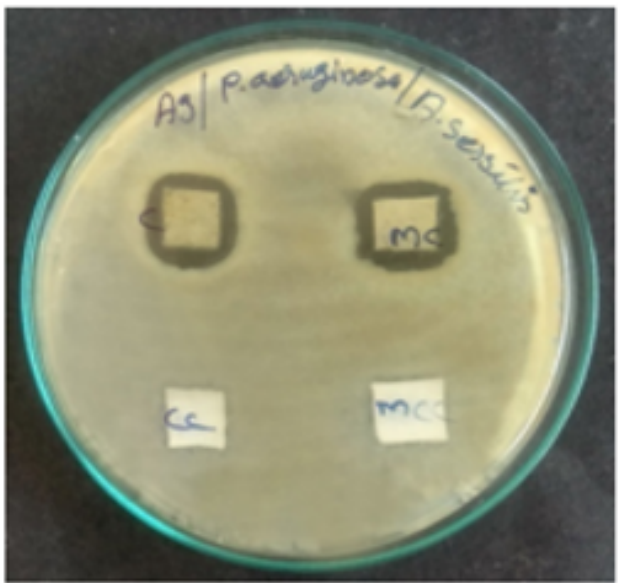

Pseudomonas aeruginosa

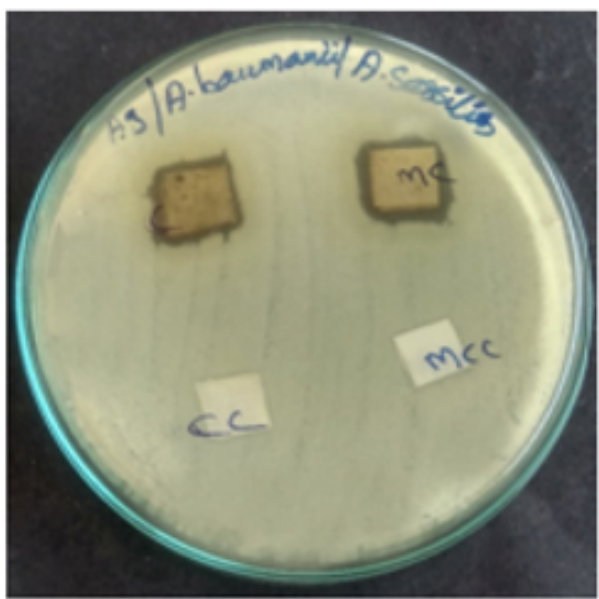

Acinetobacter baumanï

Figure 3

Antimicrobial sensitivity test of fabrics against the isolates 


\section{UV - VISIBLE SPECTROSCOPY}

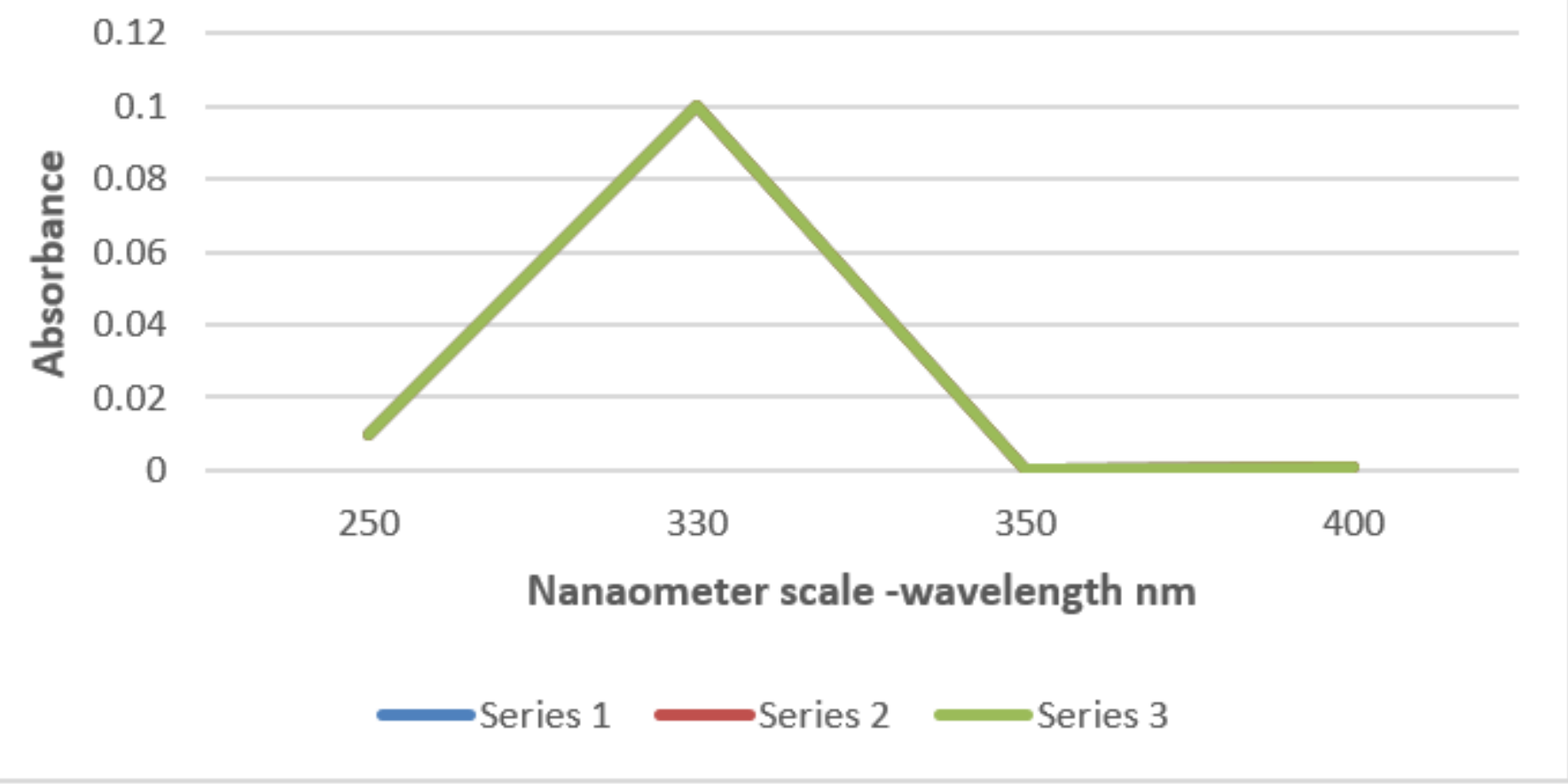

Figure 4

Spectrum of UV analysis of synthesized AS-AgNps. UV-Vis spectrum of synthesized AS-AgNPs. The uppermost absorbance apex is at $330 \mathrm{~nm}$. Note: 'A' - Absorbance, and 'B' - Wavelength (nm).

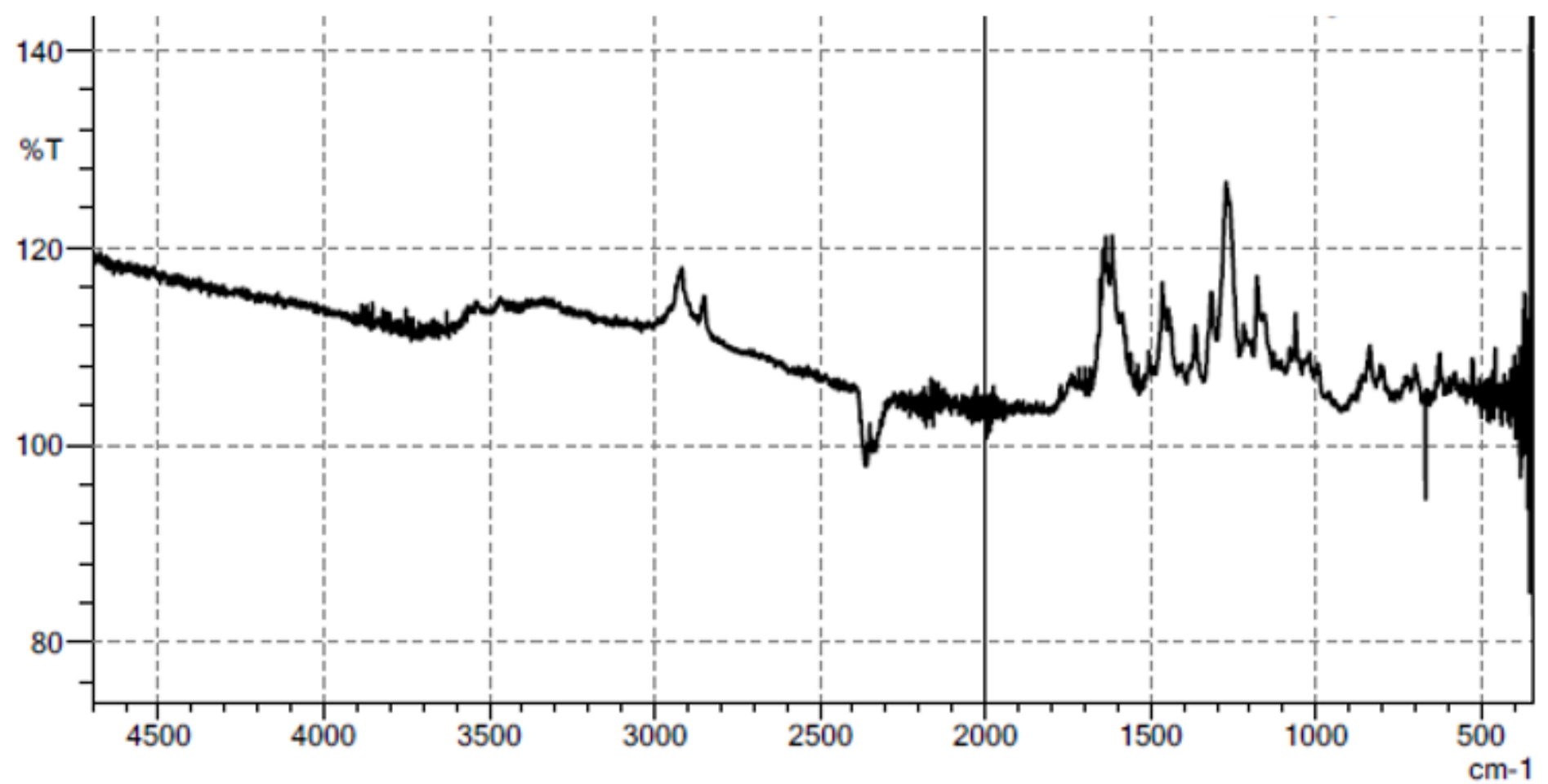

Figure 5 
Spectrum of FT-IR analysis of synthesized AS-AgNPS The synthesized AS-AgNPs exposed the subsistence of carboxylic, alkene groups, and carbonyl groups. This ultimately evinced the accessibility of plentiful chemical components embedded in the synthesized AS-AgNPs.

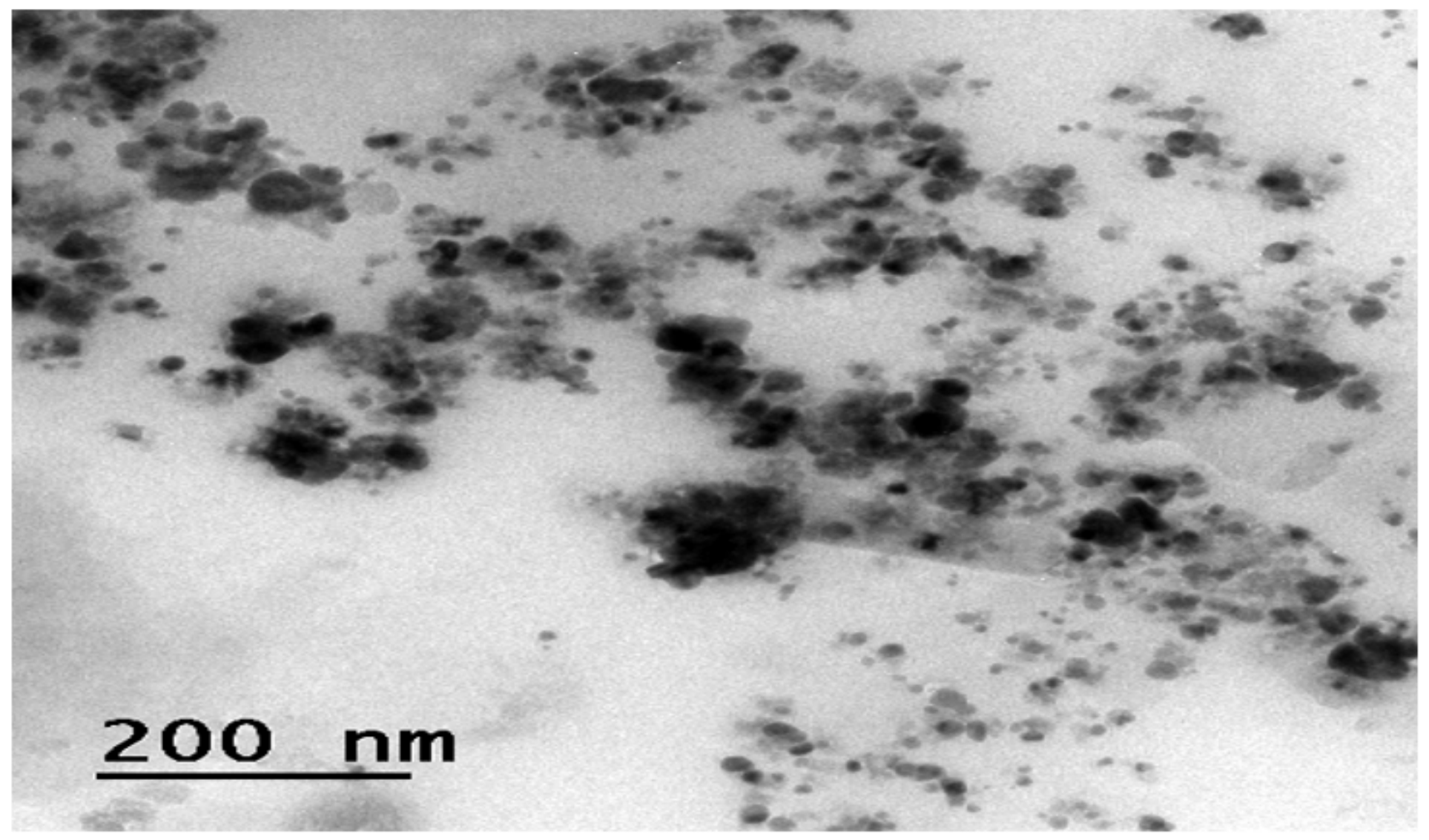

\section{Figure 6}

HR-TEM analysis of synthesized AS-AgNPs The HR-TEM investigation indicated that the synthesized ASAgNPs have the black-white crystalline, spherical, and spread in nature amid size ranging flanked by 15 and $40 \mathrm{~nm}$. 

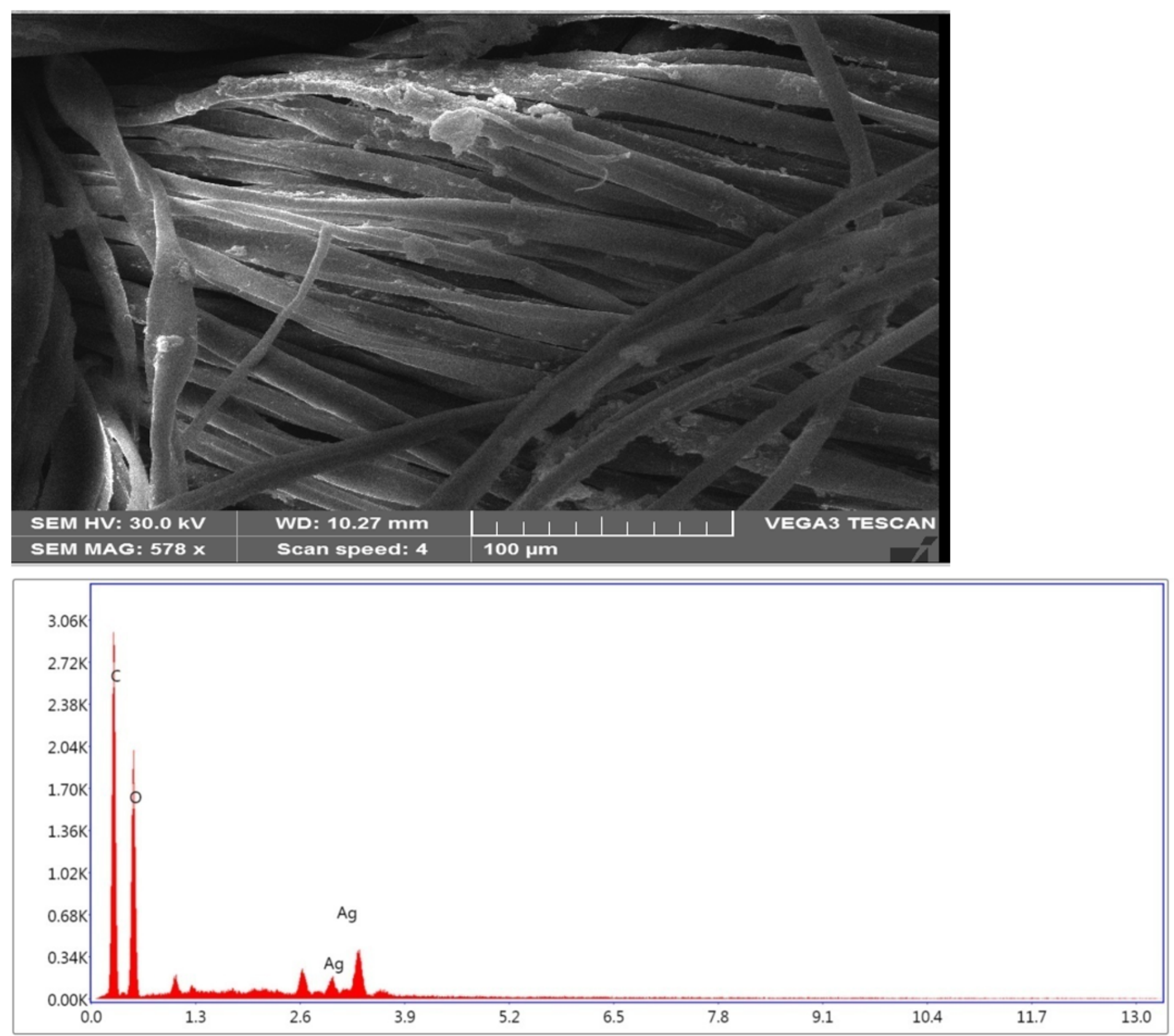

Lsec: 28.50 Cnts $0.000 \mathrm{keV}$ Det: Octane Prime Det

\section{Figure 7}

SEM and EDX analysis of AS-AgNPs coated cotton fabrics. The SEM analysis of synthesized AS-AgNPs embedded fabrics has exhibited the attachment of AS-AgNPs in the exterior of fabrics. The EDX study also evidenced the subsistence of AS-AgNPs on the fabric surface. 